\title{
Headache service quality evaluation: implementation of quality indicators in primary care in Europe
}

\author{
B. Lenz ${ }^{1,2}$ D, Z. Katsarava ${ }^{1,3,4,5^{*}}$ D, R. Gil-Gouveia ${ }^{6}$, G. Karelis ${ }^{7}$, B. Kaynarkaya ${ }^{8}$, L. Meksa ${ }^{7}$, E. Oliveira ${ }^{9}$, F. Palavra ${ }^{9,10}$, \\ I. Rosendo ${ }^{9,11}$, M. Sahin ${ }^{12}$, B. Silva ${ }^{9,13}$, D. Uludüz ${ }^{14}$, Y. Z. Ural ${ }^{15}$, I. Varsberga-Apsite ${ }^{7}$, S. T. Zengin ${ }^{16}$, L. Zvaune', \\ T. J. Steiner ${ }^{17,18}$ and on behalf of European Headache Federation and Lifting The Burden: the Global Campaign \\ against Headache
}

\begin{abstract}
Background: Lifting The Burden (LTB) and European Headache Federation (EHF) have developed a set of headache service quality indicators, successfully tested in specialist headache centres. Their intended application includes all levels of care. Here we assess their implementation in primary care.

Methods: We included 28 primary-care clinics in Germany (4), Turkey (4), Latvia (5) and Portugal (15). To implement the indicators, we interviewed 111 doctors, 92 nurses and medical assistants, 70 secretaries, 27 service managers and 493 patients, using the questionnaires developed by LTB and EHF. In addition, we evaluated 675 patients' records. Enquiries were in nine domains: diagnosis, individualized management, referral pathways, patient education and reassurance, convenience and comfort, patient satisfaction, equity and efficiency of headache care, outcome assessment and safety.
\end{abstract}

Results: The principal finding was that Implementation proved feasible and practical in primary care. In the process, we identified significant quality deficits. Almost everywhere, histories of headache, especially temporal profiles, were captured and/or assessed inaccurately. A substantial proportion (20\%) of patients received non-specific ICD codes such as R51 ("headache") rather than specific headache diagnoses. Headache-related disability and quality of life were not part of routine clinical enquiry. Headache diaries and calendars were not in use. Waiting times were long (e.g., about 60 min in Germany). Nevertheless, most patients (> 85\%) expressed satisfaction with their care. Almost all the participating clinics provided equitable and easy access to treatment, and follow-up for most headache patients, without unnecessary barriers.

(Continued on next page)

\footnotetext{
* Correspondence: zaza.katsarava@gmail.com

${ }^{1}$ Department of Neurology, Evangelical Hospital Unna, Unna, Germany ${ }^{3}$ Department of Neurology, University of Duisburg-Essen, Essen, Germany

Full list of author information is available at the end of the article
}

(c) The Author(s). 2021 Open Access This article is licensed under a Creative Commons Attribution 4.0 International License, which permits use, sharing, adaptation, distribution and reproduction in any medium or format, as long as you give appropriate credit to the original author(s) and the source, provide a link to the Creative Commons licence, and indicate if changes were made. The images or other third party material in this article are included in the article's Creative Commons licence, unless indicated otherwise in a credit line to the material. If material is not included in the article's Creative Commons licence and your intended use is not permitted by statutory regulation or exceeds the permitted use, you will need to obtain permission directly from the copyright holder. To view a copy of this licence, visit http://creativecommons.org/licenses/by/4.0/ The Creative Commons Public Domain Dedication waiver (http://creativecommons.org/publicdomain/zero/1.0/) applies to the data made available in this article, unless otherwise stated in a credit line to the data. 
(Continued from previous page)

Conclusions: The study demonstrated that headache service quality indicators can be used in primary care, proving both practical and fit for purpose. It also uncovered quality deficits leading to suboptimal treatment, often due to a lack of knowledge among the general practitioners. There were failures of process also. These findings signal the need for additional training in headache diagnosis and management in primary care, where most headache patients are necessarily treated. More generally, they underline the importance of headache service quality evaluation in primary care, not only to identify-quality failings but also to guide improvements.

This study also demonstrated that patients' satisfaction is not, on its own, a good indicator of service quality.

Keywords: Headache disorders, Headache care, Primary care, Service quality evaluation, Quality indicators, Global campaign against headache

\section{Background}

Headache disorders, although a major public-health problem, are largely treatable without need for specialist intervention, but effective care fails to reach large numbers of people worldwide [1, 2]. New headache drugs are not the answer. Although there is need for better drugs, their impact will be minimal if introduced into systems of care that cannot effectively make use of them - any more than those available already. Ten years ago, better education of medical professionals was proclaimed as the most-needed prerequisite for improving headache care [1]. In primary care management of headache, education does indeed improve practice [3].

But there is need to take a much more expansive view: the concept of service quality must take centre-stage in headache care, wherever it is delivered [4]. Service quality has broader meaning than quality of care. This and many other factors contribute to service quality, just as many causes lead to its failure.

Some years ago, as a key project within the Global Campaign against Headache [5], Lifting The Burden (LTB) and the European Headache Federation (EHF) assembled a group of experts to define quality in the context of headache care. "Good quality headache care achieves accurate diagnosis and individualized management, has appropriate referral pathways, educates patients about their headaches and their management, is convenient and comfortable, satisfies patients, is efficient and equitable, assesses outcomes and is safe" [6]. The experts analyzed and evaluated putative quality indicators, requiring that they reflect patients' and publichealth priorities and be applicable in different settings and cultures as well as to all types of headache. A total of 30 indicators were eventually assigned to nine verifiable quality domains (Table 1) [6].

In a series of evaluations, these indicators were first implemented in a pilot study in two highly specialized headache centres (at the University Hospital Essen, Germany, and the Hospital de Luz in Lisbon, Portugal) using the questionnaires developed for doctors, other health-care providers (HCPs), service managers, secretaries or administrators and patients [7]. A definitive study followed in 14 specialist-care centres, extending this evaluation across Europe [8]. Both studies found the quality indicators to be practical in specialist care and fit for purpose at this level: treatment deficits were identified and eliminated [8].

The essential next step in evaluation is to take the process into non-specialist care, including primary care, where the large majority of headache patients must be treated [4]. This study has this objective, continuing the service quality evaluation (SQE) collaborative project between LTB and EHF [5]. Its primary purpose is to assess the applicability and practical operation of the quality indicators in primary care. Its secondary purpose is to assess the quality of headache management currently in primary care in Europe, identifying deficits and providing guidance for improvement.

Table 1 The nine domains of quality in a headache service (adapted from [6])

Domain Diagnostic accuracy: appropriate enquiry; diagnoses A according to $\mathrm{IHS}$ criteria, documented during the first visit, reviewed during follow-up and supported by diagnostic diaries

Domain Individualized management: waiting time tailored to urgency; B adequate time allocation; evidence-based treatment plans reflecting diagnosis and disability, with follow-up

Domain Availability and utilization of urgent and specialist referral

C pathways

Domain Patient education and reassurance

D

Domain Convenient, clean, comfortable and welcoming service E

Domain Patient satisfaction

$\mathrm{F}$

Domain Equitable access to care, efficiency of care, cost-controls and $\mathrm{G} \quad$ avoidance of wastage

Domain Outcome measures of symptoms, disability and quality of life $\mathrm{H}$

Domain Safety of care 


\section{Methods}

\section{Ethics}

Approvals were obtained in each country in accordance with local regulations (some did not require ethics approval for studies with the primary purpose of service quality improvement). Informed consent was obtained from all study participants, regardless of whether or not ethics approval had been required.

\section{Study settings and participants}

During 2019, a total of 53 primary-care practices from four European countries were invited to participate (10 from Germany, five from Turkey, five from Latvia and 33 from Portugal), identified through personal contacts and selected to represent, as far as possible, the geographic distinctions of the four countries. Of these, 25 (47\%) declined for various reasons (commonly lack of time or interest). The study was therefore conducted in 28 (four in Germany, four in Turkey, five in Latvia and 15 in Portugal).

In each clinic we interviewed doctors (general practitioners [GPs]), other HCPs (practice nurses and/or medical assistants), service managers, secretaries and/or administrators and patients, and evaluated consecutive patients' records.

\section{Study instruments}

The data were collected prospectively under the supervision of the local principal investigator using the prescribed SQE questionnaires for each group of interviewees and for extraction of data from patients' records. The questionnaires were adapted to the primary-care setting (essentially with regard to referral pathways) and translated into the local language(s) according to LTB's translation protocols [9]. The questionnaires, in their original English, are attached in Additional Files 1, 2, 3, 4 and 5.

\section{Procedure}

In each clinic, staff were first informed of the aims and nature of the study and of the types of data to be collected, then interviewed by the local principal investigator using the appropriate questionnaires (Additional files 1, 2, 3 and 4).

All adult patients attending during the study period with the symptom "headache" were asked for interview, which proceeded with their consent. These interviews were semi-structured (Additional file 5), and conducted either by the local investigator or by GPs or other HCPs from the service trained for the purpose by the local investigator. In addition, local investigators reviewed the records of these and other randomly selected patients, extracting relevant information (Additional file 6).
Further information on the quality indicators for headache care services and their application can be found in Additional File 7.

\section{Data management and analysis}

Data were entered locally and anonymously into spreadsheets provided, and in this form transferred to the data collection centre (Clinic for Neurology, Geriatric Medicine and Neurorehabilitation in the Evangelical Hospital Unna), where they were merged and analyzed descriptively. Demographic and clinical data were provided as numerical values and summarized as percentages or mean values with standard deviations (SDs). Analyses included comparison of findings with those from 14 previously analyzed specialist-care centres [8].

No hypotheses were formulated.

Analyses and comparisons were completed in Microsoft Excel ${ }^{\circledR} 2016$.

\section{Results}

We interviewed 111 doctors, 92 nurses and medical assistants, 70 secretaries or administrators, 27 service managers and 493 patients (122 in Germany, 125 in Turkey, 156 in Latvia and 90 in Portugal), and evaluated 675 patients' records (150 in Germany, 125 in Turkey, 250 in Latvia and 150 in Portugal) (Table 2). The participating practices had similar structures but differed in size and staffing, with one to 14 doctors, one to ten other HCPs, up to two practice managers and one to seven administrative staff.

Patients were aged 18-86 years and mostly (77\%) female (Table 2). Duration of headache as the presenting complaint ranged from 1 day to 50 years, with country means varying widely from $1.9 \pm 4.6$ years in Portugal to $17.8 \pm 14.5$ years in Germany (Table 2).

Local investigators were able to collect required data quickly and efficiently from the questionnaires in all countries. All interviewed staff reported that these were easy to use and understand, and not unduly time consuming. The few comprehension difficulties (for example, "What is an outcome measure that is based on self-reported disability burden?" or "What is meant by a 'formal triage system'?") were due to lack of knowledge. These uncertainties were reflected in the limited use of outcome assessment instruments (see below).

Findings with regard to each individual quality indicator in the nine quality domains are presented by country in Table 3. The following is a narrative summary.

\section{Domain A: an accurate diagnosis is essential for optimal headache care}

Temporal profiles and specific features of presenting headaches were assessed either inaccurately or not at all in almost all practices, with Turkey the exception (88\%: 
Table 2 Characteristics of participating primary-care practices

\begin{tabular}{|c|c|c|c|c|c|c|}
\hline \multicolumn{2}{|c|}{ Practice characteristics } & \multirow{2}{*}{$\begin{array}{l}\text { Germany } \\
4\end{array}$} & \multirow{2}{*}{$\begin{array}{l}\text { Turkey } \\
4\end{array}$} & \multirow{2}{*}{$\begin{array}{l}\text { Latvia } \\
5\end{array}$} & \multirow{2}{*}{$\begin{array}{l}\text { Portugal } \\
15\end{array}$} & \multirow{2}{*}{$\frac{\text { Total }}{28}$} \\
\hline Practices & $\mathrm{n}$ & & & & & \\
\hline \multirow[t]{5}{*}{ Staff } & Doctors (GPs), n & 9 & 4 & 5 & 93 & 111 \\
\hline & Medical assistants, $\mathrm{n}$ & 15 & 0 & 3 & 0 & 18 \\
\hline & Nurses, n & 0 & 4 & 2 & 68 & 74 \\
\hline & Managers, n & 6 & 4 & 5 & 12 & 27 \\
\hline & Secretaries / administrators, $\mathrm{n}$ & 12 & 4 & 5 & 49 & 70 \\
\hline \multirow[t]{4}{*}{ Patients } & $\mathrm{n}$ & 122 & 125 & 156 & 90 & 493 \\
\hline & Female (\%) & 78 & 75 & 72 & 81 & 77 \\
\hline & Mean age $(y r \pm S D)$ & $\begin{array}{l}44.5 \pm \\
15.7\end{array}$ & $\begin{array}{l}39.7 \pm \\
14.2\end{array}$ & $\begin{array}{l}39.9 \pm \\
16.5\end{array}$ & $\begin{array}{l}45.8 \pm \\
17.8\end{array}$ & \\
\hline & Mean duration of headache $(\mathrm{yr} \pm \mathrm{SD})$ & $\begin{array}{l}17.8 \pm \\
14.5\end{array}$ & $6.9 \pm 7.5$ & $2.3 \pm 4.1$ & $1.9 \pm 4.6$ & \\
\hline $\begin{array}{l}\text { Patients' } \\
\text { records }\end{array}$ & $\mathrm{n}$ & 150 & 125 & 250 & 150 & 675 \\
\hline \multirow[t]{11}{*}{ Diagnoses } & Migraine unspecified (G43.9, G43.8), n & 54 & 47 & 28 & 24 & 153 \\
\hline & Episodic migraine without aura (G43.0), n & 12 & 0 & 1 & 0 & 13 \\
\hline & Episodic migraine with aura (G43.1), n & 6 & 1 & 4 & 0 & 11 \\
\hline & Chronic migraine (G43.3), n & 2 & 7 & 0 & 0 & 9 \\
\hline & Tension type headache (G44.2), n & 3 & 47 & 23 & 20 & 93 \\
\hline & Cluster headache (G44.0), n & 4 & 1 & 3 & 0 & 8 \\
\hline & $\begin{array}{l}\text { Other primary or secondary headache disorders (summarized under G44.8), } \\
\mathrm{n}\end{array}$ & 1 & 6 & 52 & 0 & 59 \\
\hline & Medication-overuse headache (G44.4), n & 0 & 0 & 0 & 0 & 0 \\
\hline & Mononeuritis / Occipital neuralgia (G58.8) & 9 & 0 & 0 & 0 & 9 \\
\hline & Headache unspecified (R51), n & 30 & 7 & 35 & 27 & 99 \\
\hline & Others, n & 1 & 9 & 10 & 19 & 39 \\
\hline
\end{tabular}

Table 3). About two-third of clinics documented working or definitive diagnoses at first or subsequent visits and most (92\%) reviewed these during later follow up. Diagnostic diaries were available in a minority $(0 \%$ in Germany to $30 \%$ in Latvia), with many HCPs unaware of them and others believing they were too timeconsuming. In many clinics but far from all $(45 \%$ in Turkey to $72 \%$ in Germany), diagnoses were made according to the current International Classification of Headache Disorders (ICHD) criteria; nevertheless, except in Turkey (6\%), a sizeable proportion of patients (22-30\%) received "Headache" (unspecified) diagnoses (coded R51 in ICD-10 classification) (Fig. 1).

Among specific diagnoses, migraine (whether or not further specified) was the most common in each country, followed by tension-type headache (Table 2). Latvia stood out with a high number of "Other primary or secondary headache disorders" (Table 2). No patients in any country were diagnosed with medication-overuse headache $(\mathrm{MOH})$.
Domain B: individualized management is essential for optimal headache care

Most practices did not use a formal triage system to expedite cases from waiting lists that might need this. In Germany and Portugal, the term "formal triage system" was subject to broad interpretation across interviewee groups. For example, prior to explanation of the term by the local investigator, many secretaries or administrators, but also some physicians, stated that their "triage system" was based mainly on the "trained eye" and "experience". Doctors argued that they were able to recognize "red flags", and act accordingly without a formal system for the purpose.

Patients reported mean times allocated to their visits of $16 \pm 9 \mathrm{~min}$. Across all countries, $82 \%$ of patients, on average, were satisfied with the time spent on them (Fig. 2). Patient satisfaction was by far the highest in Portugal (99\%), where the reported average visit time was longest at $24 \pm 12 \mathrm{~min}$. Nearly two thirds of HCPs, however, would have preferred more time per visit. 
Table 3 Responses to questionnaire enquiries (\% of positive answers, except where specified)

\begin{tabular}{|c|c|c|c|c|c|c|c|}
\hline $\begin{array}{l}\text { Domain and } \\
\text { indicator }\end{array}$ & $\begin{array}{l}\text { Information } \\
\text { source(s) }\end{array}$ & Enquiry & Germany & Turkey & Latvia & Portugal & Average \\
\hline A1a & Patients' records & $\begin{array}{l}\text { Is duration of presenting complaint recorded in patient's } \\
\text { record? }\end{array}$ & 43 & 98 & 20 & 51 & 47 \\
\hline$A 1 b$ & Patients' records & $\begin{array}{l}\text { Are frequency or days/month of symptoms recorded in } \\
\text { patient's record? }\end{array}$ & 25 & 88 & 8 & 30 & 32 \\
\hline $\mathrm{A} 2 \mathrm{a}$ & Patients' records & Is diagnosis recorded in patient's record? & 100 & 65 & 82 & missing & 83 \\
\hline$A 2 b$ & Patients' records & Does diagnostic record use ICHD terminology? & 72 & 46 & 53 & 41 & 53 \\
\hline A3 & Patients' records & Is working diagnosis at first visit recorded in patient's record? & 59 & 62 & 86 & 72 & 72 \\
\hline A4 & Patients' records & $\begin{array}{l}\text { Is definitive diagnosis recorded in patient's record or, if not, } \\
\text { has an appointment for review been given? }\end{array}$ & 68 & 46 & 77 & 62 & 66 \\
\hline A5 & Doctors & $\begin{array}{l}\text { Is it routine practice in your headache service to review a } \\
\text { patient's diagnosis during follow-up? }\end{array}$ & 78 & 75 & 100 & 95 & 93 \\
\hline A6 & Doctors, manager & Are diagnostic diaries available in your headache service? & 0 & 25 & 30 & 26 & 23 \\
\hline B1a & $\begin{array}{l}\text { Doctors, } \\
\text { manager, } \\
\text { secretary }\end{array}$ & Does a formal triage system exist in your headache service? & 52 & 8 & 47 & 17 & 23 \\
\hline B1b & $\begin{array}{l}\text { Doctors, } \\
\text { manager, } \\
\text { secretary }\end{array}$ & $\begin{array}{l}\text { (If yes) Is your triage system designed to pick out potentially } \\
\text { urgent cases for early appointments? }\end{array}$ & 100 & 100 & 100 & 73 & 85 \\
\hline B2a & Patients & Time per visit in minutes (mean $\pm \mathrm{SD}$ ) & $17 \pm 8$ & $8 \pm 4$ & $17 \pm 7$ & $24 \pm 12$ & $16 \pm 9$ \\
\hline $\mathrm{B} 2 \mathrm{~b}$ & Patients & Satisfaction with time per visit ("about right") & 84 & 83 & 71 & 99 & 82 \\
\hline $\mathrm{B} 2 \mathrm{C}$ & $\begin{array}{l}\text { Doctors, other } \\
\text { HCPs }\end{array}$ & $\begin{array}{l}\text { Are you satisfied that sufficient time is allocated to each } \\
\text { patient's visit to enable a good management? }\end{array}$ & 63 & 50 & 50 & 31 & 36 \\
\hline B4 & $\begin{array}{l}\text { Doctors, other } \\
\text { HCPs, manager }\end{array}$ & $\begin{array}{l}\text { Does an access route to psychological therapies exist in your } \\
\text { headache service? }\end{array}$ & 67 & 33 & 33 & 67 & 63 \\
\hline B5 & $\begin{array}{l}\text { Doctors, other } \\
\text { HCPs, manager }\end{array}$ & $\begin{array}{l}\text { Is an instrument for disability assessment available in your } \\
\text { headache service? }\end{array}$ & 7 & 8 & 33 & 21 & 19 \\
\hline B6a & $\begin{array}{l}\text { Doctors, other } \\
\text { HCPs, manager }\end{array}$ & $\begin{array}{l}\text { Does your headache service allow follow-up of every patient } \\
\text { who needs it? }\end{array}$ & 97 & 67 & 100 & missing & 91 \\
\hline B6b & $\begin{array}{l}\text { Doctors, other } \\
\text { HCPs, manager }\end{array}$ & $\begin{array}{l}\text { Is a follow up diary or calendar available in your headache } \\
\text { service? }\end{array}$ & 23 & 25 & 40 & 24 & 25 \\
\hline $\mathrm{Cl}$ & $\begin{array}{l}\text { Doctors, } \\
\text { manager, } \\
\text { secretary }\end{array}$ & Does a referral pathway exist? & 85 & 0 & 33 & 90 & 80 \\
\hline $\mathrm{C} 2$ & $\begin{array}{l}\text { Doctors, } \\
\text { manager, } \\
\text { secretary }\end{array}$ & $\begin{array}{l}\text { (If yes) Does this pathway permit, and respond to, urgent } \\
\text { referral when needed? }\end{array}$ & 83 & 0 & 100 & 83 & 84 \\
\hline D1a & $\begin{array}{l}\text { Doctors, other } \\
\text { HCPs, manager }\end{array}$ & Are information leaflets available? & 27 & 0 & 60 & 14 & 18 \\
\hline D1b & Patients & Doctor provides patient with information & 48 & 81 & 96 & 99 & 81 \\
\hline D1c & Patients & (If yes) Information given understandable? & 100 & 97 & 92 & 99 & 96 \\
\hline D1d & Patients & (If yes) Amount of information about right & 83 & 88 & 67 & 94 & 81 \\
\hline D2 & Patients & Patients were given reassurance & 91 & 82 & 86 & 97 & 88 \\
\hline E1a & Patients & Service environment clean and comfortable & 98 & 82 & 96 & 98 & 93 \\
\hline E1b & $\begin{array}{l}\text { Doctors, other } \\
\text { HCPs }\end{array}$ & Service environment clean and comfortable & 88 & 75 & 90 & 43 & 52 \\
\hline E2 & Patients & Satisfaction with welcome & 99 & 96 & 90 & 100 & 96 \\
\hline E3a & Patients & $\begin{array}{l}\text { How long were you kept waiting to see the doctor (in } \\
\text { minutes)? (mean } \pm \text { SD) }\end{array}$ & $57 \pm 53$ & $6 \pm 7$ & $\begin{array}{l}15 \pm \\
15\end{array}$ & $24 \pm 28$ & $24 \pm 36$ \\
\hline E3b & $\begin{array}{l}\text { Doctors, other } \\
\text { HCPs }\end{array}$ & Satisfaction with waiting time & 67 & 100 & 100 & 85 & 84 \\
\hline E3c & Patients & Satisfaction with waiting time & & & & & \\
\hline
\end{tabular}


Table 3 Responses to questionnaire enquiries (\% of positive answers, except where specified) (Continued)

\begin{tabular}{|c|c|c|c|c|c|c|c|}
\hline $\begin{array}{l}\text { Domain and } \\
\text { indicator }\end{array}$ & $\begin{array}{l}\text { Information } \\
\text { source(s) }\end{array}$ & Enquiry & Germany & Turkey & Latvia & Portugal & Average \\
\hline & & reasonable & 60 & 75 & 79 & 83 & 74 \\
\hline & & too long & 23 & 17 & 16 & 15 & 18 \\
\hline & & much too long & 17 & 8 & 5 & 2 & 8 \\
\hline \multirow[t]{6}{*}{ F1 } & Patients & Satisfaction with overall management & & & & & \\
\hline & & very good & 38 & 9 & 9 & 26 & 19 \\
\hline & & good & 39 & 36 & 40 & 28 & 37 \\
\hline & & adequate & 15 & 46 & 37 & 45 & 35 \\
\hline & & poor & 7 & 7 & 13 & 1 & 8 \\
\hline & & very poor & 1 & 2 & 1 & 0 & 1 \\
\hline G1 & Manager & Protocol to limit wastage exists & 33 & 25 & 20 & 42 & 33 \\
\hline G2 & Manager & Record of input costs exists & 83 & 0 & 0 & 58 & 44 \\
\hline G3 & $\begin{array}{l}\text { Doctors, other } \\
\text { HCPs, manager }\end{array}$ & Policy to ensure equal access exists & 100 & 67 & 93 & 81 & 83 \\
\hline $\mathrm{H} 1$ & $\begin{array}{l}\text { Doctors, other } \\
\text { HCPs, manager }\end{array}$ & $\begin{array}{l}\text { Outcome measures include Headache Under-Response to } \\
\text { Treatment questionnaire (HURT) or similar }\end{array}$ & 10 & 25 & 13 & 5 & 7 \\
\hline $\mathrm{H} 2$ & $\begin{array}{l}\text { Doctors, other } \\
\text { HCPs, manager }\end{array}$ & $\begin{array}{l}\text { Outcome measures include Headache-Attributed Lost Time } \\
\text { index (HALT) or similar }\end{array}$ & 0 & 8 & 0 & 3 & 3 \\
\hline $\mathrm{H} 3$ & $\begin{array}{l}\text { Doctors, other } \\
\text { HCPs, manager }\end{array}$ & $\begin{array}{l}\text { Outcome measures include World Health Organization Quality } \\
\text { of Life questionnaire (WHOQoL) or similar }\end{array}$ & 3 & 0 & 13 & 3 & 4 \\
\hline 11 & $\begin{array}{l}\text { Doctors, other } \\
\text { HCPs, manager }\end{array}$ & Protocol for reporting serious adverse events exists & 73 & 25 & 80 & 58 & 60 \\
\hline
\end{tabular}

One third of practices in Latvia, but fewer elsewhere, used an instrument for disability assessment at the time of diagnosis. However, in Germany, the recording of sick days or sick leave due to a recurring diagnosis was understood as a record of disability.

In Germany and Portugal, two thirds of practices had an access route to psychological therapies, but in Turkey and Latvia only one-third. In this context it should be mentioned that, in Germany in particular, many GPs stated that they themselves could offer psychotherapeutic or psychological treatments to their patients on account of their additional training or qualifications.

Almost all practices were able to offer their patients follow-up, when considered necessary, although few used standardized follow-up diaries or outcome assessment instruments to monitor progress.

Domain C: appropriate referral pathways are essential for optimal headache care

In Germany and Portugal, more than $85 \%$ of HCPs indicated that appropriate onward referral pathways existed, but in Latvia only one third and in Turkey none. In Germany, the destination of referrals was largely determined by long waiting times (often several weeks to months) for outpatient specialist care, so this was not an option for time-critical referrals. Neurologists' waiting times were sharply criticized by both patients and HCPs.
Extended waiting times for elective hospital admissions were also criticized, with the result that admissions were often made via the emergency room.

\section{Domain D: education of patients about their headache and their management is essential for optimal headache care}

As with diagnostic questionnaires and diaries, few HCPs in most countries were aware that patient information leaflets were freely available in various languages. Accordingly, except in Latvia, information leaflets were not often available. Nevertheless, the great majority of patients expressed satisfaction with the information and reassurance provided by their doctors.

\section{Domain E: convenience and comfort are part of the optimal headache care}

More patients than HCPs considered their service environment to be clean and comfortable, most markedly in Portugal (HCPs 43\%, patients 98\%). Almost all patients felt welcomed. Reported waiting times varied widely (mean $24 \pm 36 \mathrm{~min}$ ), being least in Turkey $(6 \pm 7 \mathrm{~min}$ ) and longest by far in Germany ( $57 \pm 53 \mathrm{~min}$ ); nevertheless, only a minority of patients and HCPs were dissatisfied with waiting times, and nearly $60 \%$ of German patients found them acceptable (Fig. 2). 


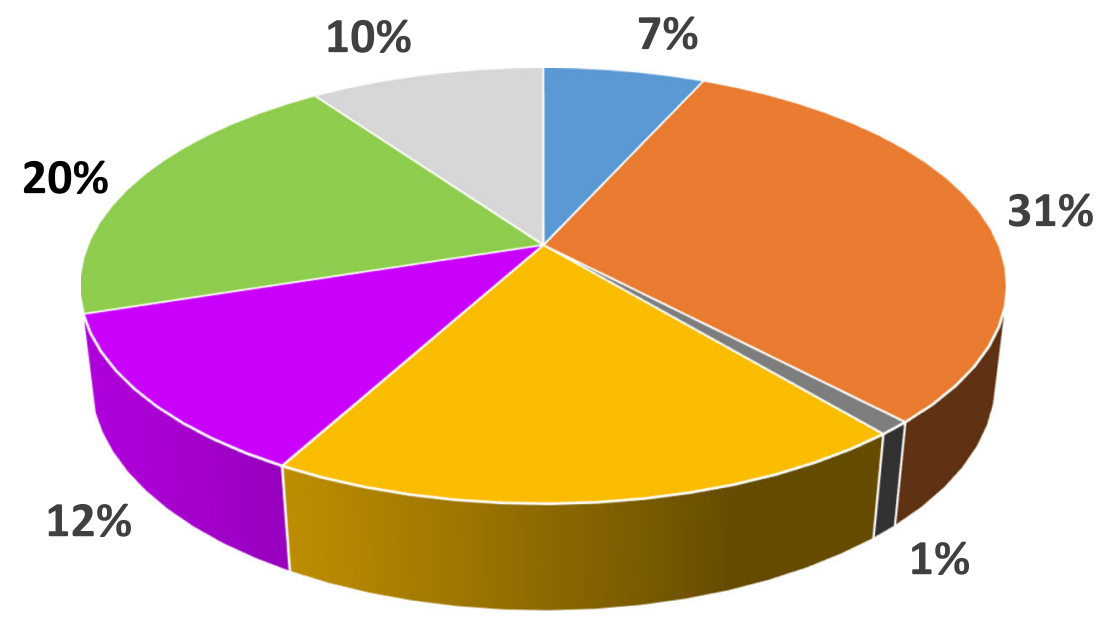

$19 \%$

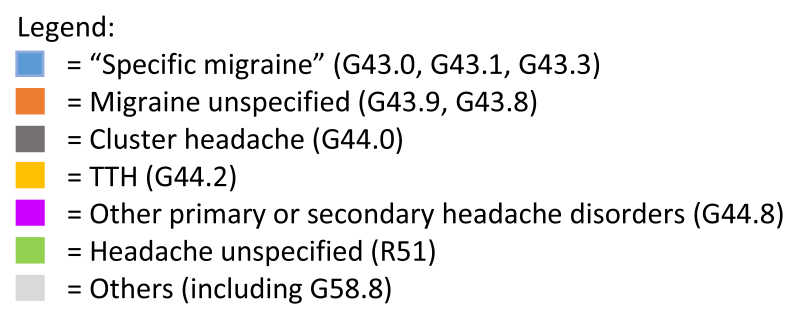

Fig. 1 Distribution of diagnoses across practices (percent)

Domain F: achieving patient satisfaction is part of optimal headache care

Overall patient satisfaction was high in all countries, 91\% rating their management adequate, good or very good (Fig. 2).

\section{Domain G: optimal headache care is efficient and equitable}

About one-third of practices had protocols to avoid wastage of resources. Running costs were recorded in Germany (83\%) and in Portugal (58\%), but not in Turkey or Latvia. Most practices (83\%, but only 67\% in Turkey) offered equal access to their (headache) service for all patients.

\section{Domain H: outcome assessment is essential in optimal headache care}

Outcome assessment instruments (e. g. HURT, HALT, WHOQoL) were not in use routinely in primary care. In personal conversations, it was found that the majority of HCPs in Germany were not aware of these questionnaires or other options. Headache intensity was assessed by Visual Analogue Scale (VAS). In addition in Germany, change in frequency of sick leave attributed to a long-term diagnosis served as a disability indicator and as a measure of treatment outcome.

Domain I: optimal headache care is safe

On average nearly $60 \%$ of all general practices had formal protocols to ensure reporting of serious adverse events. Turkey (25\%) was unusual in this aspect of care.

\section{Comparisons between specialist-care centres and primary-care practices in Europe}

We made comparisons between the 28 primary-care practices and 14 specialized-care centres, using data collected several years ago in university-based headache clinics throughout Europe [8]. Figure 3 shows these in eight quality domains (excluding referral pathways, which have different implications in primary and specialist care). Quality of headache care was inferior in primary care on almost all indicators in all domains. The most marked differences could be identified in domain A (Accurate diagnosis) and domain H (Outcome assessment), but there were notable deficits in primary care relative to specialist care in other individual quality indicators (for example in the availability of information leaflets [D1a]). 

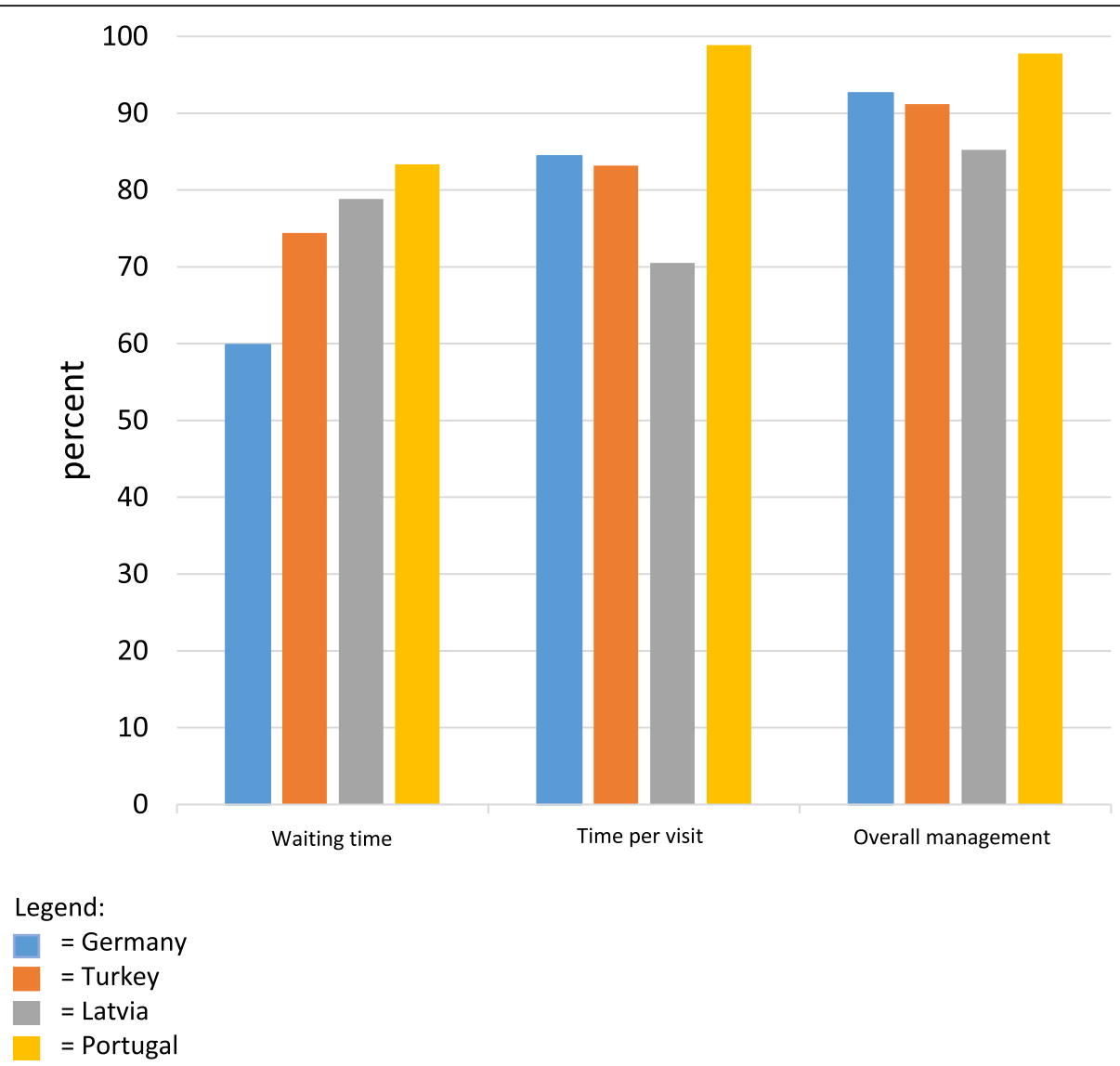

Fig. 2 Patients' satisfaction with three aspects of their care, by country

Primary care was, however, superior in providing equitable and easy access to headache care. Furthermore, the vast majority of patients were satisfied with their headache care in both primary and specialist care.

\section{Discussion}

To our knowledge, this was the first study addressing quality of headache care in primary care. It continued and was an important extension of the evaluation process of the quality indicators for headache care developed by LTB and EHF; previous studies were conducted in specialist centres $[7,8]$. Although the participating practices differed in size, staffing levels and the national health-care systems in which they were set, findings were broadly comparable between countries and common trends in practice were evident. The essential findings related to practicality of the indicators, and their fitness for purpose in primary care. Regarding the former, all interviewed staff found the questionnaires easy to use and understand, and, crucially, not unduly time consuming. As to the latter, this was evidenced in the assessments made. It was not a primary purpose of this study to assess service quality in primary care but, in doing so, the indicators demonstrated their fitness for purpose.

This was best illustrated in the comparisons between primary and specialized care: by most indicators in all quality domains, specialized headache clinics performed better than primary care. Any other finding would have cast serious doubt on the validity of the indicators. As it is, the comparisons demonstrated that the indicators can be used in different settings with the expectation of revealing quality differences. We note here that, while the indicators identify quality deficits, they do not yet determine whether or not quality is "good" in any particular setting. Future benchmarking studies are needed for this.

Among specific indicators, lack of formal triage systems was a not unexpected feature of primary care, to which, unlike specialist care, access is generally unrestricted and not subject to long and potentially harmful delays. Doctors' assertions seemed reasonable that experience enabled triage in primary care without a formal system for the purpose. This aside, in all countries, quality deficits were uncovered, and these led inevitably to suboptimal treatment judged objectively - although this was not reflected in patients' satisfaction ratings (we say more on this later). Most deficits were attributable to 

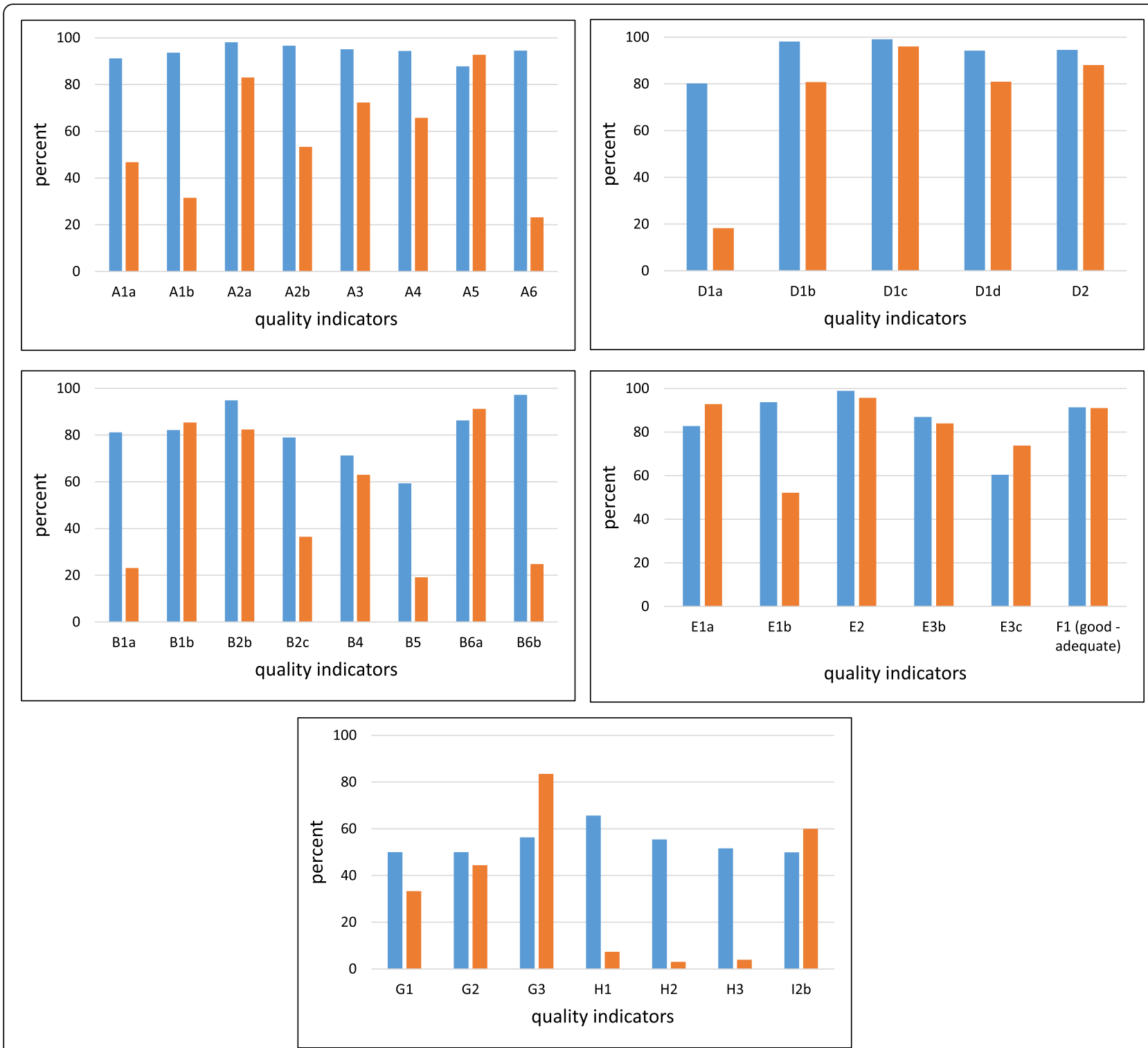

Legend:

$\square=14$ specialist-care centres in Europe

$\square=28$ primary-care practices in Europe

Fig. 3 Headache senvice quality evaluation: comparisons between specialist and primary care in Europe in eight quality domains. A: accurate diagnosis; B: individualized management; D: education of patients; E: convenience and comfort; F: patients' satisfaction; G: efficiency and equitability; H: outcome assessment; I: safety

HCPs' lack of relevant knowledge. While this might also, to an extent, be expected in non-specialist care, it needs remediation given that headache disorders are the most common single cause of consultation in primary care $[10,11]$. Education of HCPs in headache was identified 10 years ago by WHO and LTB as the most pressing need, worldwide, in the pursuit of better headache care [1]. Basic but structured education of GPs has been shown to improve their practice, significantly increasing proportions of patients given specific diagnoses and treatments, both indicators of headache care quality $[3,12]$.

Pertaining to this, an important particular finding was that a substantial proportion of patients of these primary-care practices received non-specific ICD codes such as R51 ("headache") rather than specific headache diagnoses. To be fair, coding was often driven by practical rather than clinical considerations, or by administrative requirements, and might not reflect diagnostic deficiencies. Nevertheless, almost everywhere, accurate 
diagnosis was impeded by inadequately assessed histories of headache, especially with regard to temporal profiles, unaided by simple aids such as headache diaries and calendars. It is well recognized that the history is allimportant in correct headache diagnosis, itself the essential foundation of successful treatment [13], and unsurprising therefore that the Eurolight study has already demonstrated headache management to be generally suboptimal in European primary care [14]. A particularly telling finding in our study was that none of the practices gave a single diagnosis of $\mathrm{MOH}$, despite that $\mathrm{MOH}$ is common in the general population level (prevalence ranging between $0.5 \%$ and $7.2 \%[15]$ ) and that people with $\mathrm{MOH}$ are highly likely to seek GP consultation. Another was Latvia's high number of "Other primary or secondary headache disorders", which could be summarized under ICD-10 code G44.8 and included "Headache attributed to arterial hypertension" (ICHD-3 code 10.3, $n=29$ ), "Alcohol-induced headache" (ICHD-3 code 8.14, $n=7)$ and "Headache attributed to acute rhinosinusitis" (ICHD-3 code 11.5, $n=4$ ). While Germany, Turkey and Portugal might have subsumed these under R51, or coded them only according to the underlying causative disorders, there is reason to question these. In particular, ICHD notes that the first of them is attributable only to severe hypertension and is uncommon [16], but in some countries a contrary belief persists.

Although it was not a primary purpose, our study emphasizes the need, in all countries studied, for wider implementation of educational programmes aimed at GPs.

Good management of headache patients begins with an explanation of their disorder and of the purpose and means of management [12]. This is time consuming. Time constraints were mentioned by doctors in all countries as a key factor impeding good management. Time can be saved, and good management supported, by offering patients information leaflets, such as those produced by LTB and freely available [13]. Every patient offered a treatment or whose treatment is changed requires follow-up in order to assess its success against expectation [13]. This, also, is time consuming, but can be aided by various outcome measurement instruments, again freely available $[17,18]$. None of these were in routine use in the primary-care practices. While this was largely because of lack of awareness of them, some GPs argued that time constraints themselves made it impossible to incorporate them into everyday routine. This argument is mistaken: patients can fill in questionnaires at home, or in the waiting room, so that use of these kinds of instrument is time saving. Education can solve this, while introducing these aids into primary-care headache management is a low-cost intervention.

There were some positive findings for primary care. Most importantly, the great majority of the primary-care services provided equitable and easy access to headache care, without barriers, and were able to provide followup to every patient who needed it. These essential features of primary care $[19,20]$ distinguished it sharply from specialized care. Furthermore, patients overall were satisfied with their care: with the time allocated to them, with the care environment and with their management overall. There are three points to be made here. First, these evaluations reflect the common trust of patients in family doctors all over the world [19, 21-24], which is at the heart of good patient-doctor interaction. Second, they show the importance attributed by patients to structure and process [6,25-28]. Third, and of particular significance in this context, is that patients' satisfaction, while essential as a quality indicator, is unreliable on its own. There may be multiple reasons for this, but these are beyond the scope of this paper.

It should be noted that the quality indicators do not assess outcomes themselves. The original developers of the indicators acknowledged the impossibility of this: it would require parallel prospective individual patient assessments and follow-up, a process beset by ethical difficulties as well as high risk of changing observed practices (Hawthorne effect) $[6,29]$. The expectation underlying $\mathrm{SQE}$ that is primarily focused on structure and process [25], as these indicators are, is that structure and process are drivers of outcomes: good promote good, and poor lead inevitably to poor.

While the study fulfilled its primary goal of demonstrating that the SQE methodology is applicable and practical in primary care, understandable to HCPs and patients without being unduly time consuming, we note a limitation and caveat in regard to this: only $53 \%$ of invited primary-care clinics agreed to participate. This was a consequence of the very reasonable reluctance of GPs to engage in research in an area not of special interest to them, but it resulted in a potential selection bias. This ought not to restrict the generalisability of the finding: while some practices might be less willing than others to accept the intrusion of SQE, this will be feasible methodology in all those that do. The study was also limited in its inclusion of only four countries, each represented to different extents (from four practices in Germany and Turkey to 15 in Portugal, with varying numbers of participating staff and patients), but, within Europe, these are diverse countries. Despite that it was not the study's primary purpose, it uncovered opportunities for improvement in the management of headache patients in primary care. The quality indicators, as well as identifying-quality failings, can guide improvements.

\section{Conclusion}

This study, the first evaluating headache service quality indicators in primary care in Europe, confirmed the 
indicators to be practical and fit for purpose, able to identify areas for improvement in pursuit of care quality. While quality criteria must be deployed at all levels within health-care-systems (and this study has confirmed that they can be), primary care is the setting of greatest importance since it is where management of the majority of headache-patients can and should be based [1, 4, 13]. This study, in the context of the collaborative LTB/ EHF SQE project of which it is part, is a step towards bringing headache service quality centre-stage.

The next step is benchmarking studies, defining reasonable expectations of quality, and what constitutes "good" in this context, in each setting.

\section{Abbreviations}

EHF: European Headache Federation; GP: General practitioner; HALT: Headache-Attributed Lost Time (index\}; HCP: Health-care provider; HURT: Headache Under-Response to Treatment (questionnaire); ICD: International Statistical Classification of Diseases and Related Health Problems; ICHD: International Classification of Headache Disorders; LTB: Lifting The Burden; $\mathrm{MOH}$ : Medication-overuse headache; SD: Standard deviation; VAS: Visual Analogue Scale; WHO: World Health Organization; WHOQOL: WHO quality of life (questionnaire)

\section{Supplementary Information}

The online version contains supplementary material available at https://doi. org/10.1186/s10194-021-01236-4.

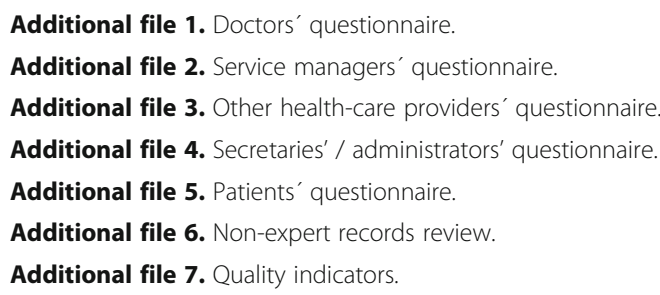

\section{Acknowledgments}

This study was promoted by the European Headache Federation and by Lifting The Burden, a UK-registered non-governmental organization conducting the Global Campaign against Headache in official relations with the World Health Organization.

\section{Authors' contributions}

TJS and ZK conceived the SQE project of which this was part. ZK and RG-G conceived this study and developed the protocol. ZK, RG-G, GK, BK, LM, EO, $F P, I R, M S, B S, D U, Y Z U, I V-A, S T Z$ and LZ recruited primary-care practices and oversaw data collection. BL performed the analyses and, with TJS, drafted the manuscript. All authors read, contributed to and approved the final manuscript.

\section{Funding}

The German part was supported by Novartis ${ }^{\oplus}$, without influence on design, conduct, analysis or reporting. Other parts received no financial support.

\section{Availability of data and materials}

The national data sets generated by this study are not publicly accessible but are available for legitimate purposes upon request from the respective authors.

\section{Declarations}

\section{Ethics approval and consents to participate}

Ethics approvals were obtained in accordance with national requirements: from the Ethics Commission of the Westphalia-Lippe Medical Association and the Westphalian Wilhelm University of Münster for the German part of this study and from the Centre Regional Health Administration Ethics Committee (ARS Centro, Portugal) for the Portuguese part. Approvals were not required in Turkey or Latvia for projects with the primary purpose of service improvement.

\section{Consent for publication \\ Not applicable.}

\section{Competing interests}

TJS and ZK are Directors and Trustees of Lifting The Burden. ZK is President of the European Headache Federation. Other than these, no author has competing interests relevant to the subject matter of this manuscript.

\section{Author details \\ ${ }^{1}$ Department of Neurology, Evangelical Hospital Unna, Unna, Germany. ${ }^{2}$ Department of Neurology, Bundeswehr Central Hospital Koblenz, Koblenz, Germany. ${ }^{3}$ Department of Neurology, University of Duisburg-Essen, Essen, Germany. ${ }^{4}$ EVEX Medical Corporation, Tbilisi, Republic of Georgia. ${ }^{5}$ IM Sechenov First Moscow State Medical University (Sechenov University), Moscow, Russian Federation. ${ }^{6}$ Hospital da Luz Headache Center, Lisbon, Portugal. ${ }^{7}$ Riga East Clinical University Hospital, Neurology and Neurosurgery Department, Headache Unit, Riga, Latvia. ${ }^{8}$ Kagıthane Yahya Kemal ASM, Istanbul, Turkey. ${ }^{9}$ Faculty of Medicine, University of Coimbra, Coimbra, Portugal. ${ }^{10}$ Centre for Child Development - Neuropediatrics Unit, Hospital Pediátrico, Centro Hospitalar e Universitário de Coimbra, Coimbra, Portugal. \\ ${ }^{11}$ Family Health Unit "Coimbra Centro", Coimbra, Portugal. ${ }^{12}$ Kartal 10 Nolu ASM Istanbul, Istanbul, Turkey. ${ }^{13}$ Family Health Unit "Pulsar", Coimbra, Portugal. ${ }^{14}$ Neurology Department, Istanbul University Cerrahpasa School of Medicine, Istanbul, Turkey. ${ }^{15}$ Esenler Havaalanı ASM, Istanbul, Turkey. \\ ${ }^{16}$ Bagcılar Yıldıztepe ASM, Istanbul, Turkey. ${ }^{17}$ Norwegian University of Science and Technology, Trondheim, Norway. ${ }^{18}$ Division of Neuroscience, Imperial College London, London, UK.}

Received: 3 February 2021 Accepted: 31 March 2021

Published online: 28 April 2021

\section{References}

1. World Health Organization and Lifting The Burden (2011) Atlas of headache disorders and resources in the world 2011. World Health Organization, Geneva

2. Steiner TJ, Birbeck GL, Jensen $R H$, Katsarava Z, Stovner LJ, Martelletti $P$ (2015) Headache disorders are third cause of disability worldwide. J Headache Pain 16(1):58. https://doi.org/10.1186/s10194-015-0544-2

3. Braschinsky M, Haldre S, Kals M, lofik A, Kivisild A, Korjas J, Koljal S, Katsarava Z, Steiner TJ (2016) Structured education can improve primary-care management of headache: the first empirical evidence, from a controlled interventional study. J Headache Pain 17(1):24. https://doi.org/10.1186/s101 94-016-0613-1

4. Steiner TJ, Antonaci F, Jensen R, Lainez MJ, Lanteri-Minet M, Valade D, European Headache Federation, Global Campaign againist Headache (2011) Recommendations for headache service organisation and delivery in Europe. J Headache Pain 12(4):419-426. https://doi.org/10.1007/s10194-0110320-X

5. Steiner TJ (2005) Lifting The Burden: the global campaign to reduce the burden of headache worldwide. J Headache Pain 6(5):373-377. https://doi. org/10.1007/s10194-005-0241-7

6. Peters $M$, Jenkinson C, Perera S, Loder E, Jensen R, Katsarava Z, Gil Gouveia R, Broner S, Steiner T (2012) Quality in the provision of headache care. 2: defining quality and its indicators. J Headache Pain 13(6):449-457. https:// doi.org/10.1007/s10194-012-0465-2

7. Katsarava Z, Gil Gouveia R, Jensen R et al (2015) Evaluation of headache service quality indicators: pilot implementation in two specialist-care centres. J Headache Pain 16(1):537. https://doi.org/10.1186/s10194-015-0537-1

8. Schramm S, Uluduz D, Gouveia RG, Jensen R, Siva A, Uygunoglu U, Gvantsa G, Mania M, Braschinsky M, Filatova E, Latysheva N, Osipova V, 
Skorobogatykh K, Azimova J, Straube A, Eren OE, Martelletti P, de Angelis V, Negro A, Linde M, Hagen K, Radojicic A, Zidverc-Trajkovic J, Podgorac A, Paemeleire K, de Pue A, Lampl C, Steiner TJ, Katsarava Z (2016) Headache service quality: evaluation of quality indicators in 14 specialist-care centres. J Headache Pain 17(1):111. https://doi.org/10.1186/s10194-016-0707-9

9. Steiner TJ, Paemeleire K, Jensen $R$ et al (2007) Aids for management of common headache disorders in primary care. J Headache Pain 8(S1):1-47. https://doi.org/10.1007/s10194-007-0428-1

10. Zentralinstitut für die kassenärztliche Versorgung in Deutschland (Berlin, 2016) Die 50 häufigsten ICD-10-Schlüsselnummern nach Fachgruppen: aus dem ADT-Panel des Zentralinstituts

11. Torres RCS, Marques KS, Leal KNR et al (2015) Main reasons for medical consultations in family healthcare units in the city of Recife, Brazil: a crosssectional study. Sao Paulo Med J 133(4):367-370. https://doi.org/10.1590/1 516-3180.2014.9490902

12. Braschinsky M, Haldre S, Kals M, Arge M, Saar B, Niibek M, Katsarava Z, Steiner TJ (2018) Structured education to improve primary-care management of headache: how long do the benefits last? A follow-up observational study. Eur J Neurol 25(3):497-502. https://doi.org/10.1111/ ene. 13524

13. Steiner $T$, Jensen $R$, Katsarava Z, Linde M, MacGregor EA, Osipova V, Paemeleire K, Olesen J, Peters M, Martelletti P (2019) Aids to management of headache disorders in primary care (2nd edition): on behalf of the European headache federation and Lifting The Burden: the global campaign against headache. J Headache Pain 20(1):57. https://doi.org/10.1186/s10194018-0899-2

14. Katsarava Z, Mania M, Lampl C, Herberhold J, Steiner TJ (2018) Poor medical care for people with migraine in Europe - evidence from the Eurolight study. J Headache Pain 19(1):10. https://doi.org/10.1186/s10194-018-0839-1

15. Vandenbussche N, Laterza D, Lisicki M, Lloyd J, Lupi C, Tischler H, Toom K, Vandervorst F, Quintana S, Paemeleire K, Katsarava Z (2018) Medicationoveruse headache: a widely recognized entity amidst ongoing debate. J Headache Pain 19(1):50. https://doi.org/10.1186/s10194-018-0875-x

16. Headache Classification Committee of the International Headache Society (2018) The international classification of headache disorders, 3rd edition. Cephalalgia 38(1):1-211. https://doi.org/10.1177/0333102417738202

17. Steiner TJ, Buse DC, Al Jumah M et al (2018) The headache under-response to treatment (HURT) questionnaire, an outcome measure to guide follow-up in primary care: development, psychometric evaluation and assessment of utility. J Headache Pain 19(1):15. https://doi.org/10.1186/s10194-018-0842-6

18. Steiner TJ, Lipton RB (2018) The headache-attributed lost time (HALT) indices: measures of burden for clinical management and population-based research. J Headache Pain 19(1):12. https:/doi.org/10.1186/s10194-018-0837-3

19. World Health Organization Declaration of Alma-Ata: International Conference on Primary Health Care, Alma-Ata, USSR, 6-12 September 1978

20. World Health Organization (2019) Primary health care: Key facts. https:// www.who.int/news-room/fact-sheets/detail/primary-health-care. Accessed 08 Dec 2020

21. Pearson SD, Raeke LH (2000) Patients' Trust in Physicians: many theories, few measures, and little data. J Gen Intern Med 15(7):509-513. https://doi. org/10.1046/j.1525-1497.2000.11002.x

22. Banerjee A, Sanyal D (2012) Dynamics of doctor-patient relationship: a crosssectional study on concordance, trust, and patient enablement. J Family Community Med 19(1):12-19. https://doi.org/10.4103/2230-8229.94006

23. Mainous AG III, Baker R, Love MM et al (2001) Continuity of Care and Trust in One's Physician: Evidence From Primary Care in the United States and the United Kingdom. Special Article: Distinguished Paper From the 2000 North American primary care research group meeting. Fam Med. 2001;33(1):22-27.

24. Crocker JE, Swancutt DR, Roberts MJ et al (2013) Factors affecting patients trust and confidence in GPs: evidence from the English national GP patient survey. BMJ Open 3(5):1-8. https://doi.org/10.1136/bmjopen-2013-002762

25. Donabedian A (1988) The quality of care: how can it be assessed? J Am Med Assoc 260(12):1743-1748. https://doi.org/10.1001/jama.1988.0341012 0089033

26. Kleij K-S, Tangermann U, Amelung VE, Krauth C (2017) Patients' preferences for primary health care - a systematic literature review of discrete choice experiments. BMC Health Serv Res 17(1):476. https://doi.org/10.1186/s12913017-2433-7

27. Krinke K-S, Tangermann U, Amelung VE, Krauth C (2019) Public preferences for primary care provision in Germany - a discrete choice experiment. BMC Fam Pract 20(1):80. https://doi.org/10.1186/s12875-019-0967-y
28. Cheraghi-Sohi S, Bower P, Mead N, McDonald R, Whalley D, Roland M (2006) What are the key attributes of primary care for patients? Building a conceptual 'map' of patient preferences. Health Expect 9(3):275-284. https:// doi.org/10.1111/j.1369-7625.2006.00395.x

29. Peters M, Perera S, Loder E, Jenkinson C, Gil Gouveia R, Jensen R, Katsarava Z, Steiner TJ (2012) Quality in the provision of headache care. 1: systematic review of the literature and commentary. J Headache Pain 13(6):437-447. https://doi.org/10.1007/s10194-012-0466-1

\section{Publisher's Note}

Springer Nature remains neutral with regard to jurisdictional claims in published maps and institutional affiliations.
Ready to submit your research? Choose BMC and benefit from:

- fast, convenient online submission

- thorough peer review by experienced researchers in your field

- rapid publication on acceptance

- support for research data, including large and complex data types

- gold Open Access which fosters wider collaboration and increased citations

- maximum visibility for your research: over $100 \mathrm{M}$ website views per year

At BMC, research is always in progress.

Learn more biomedcentral.com/submissions 